\title{
UM ROLE PLAYING GAME PARA O ENSINO DE ELICITAÇÃO E ANÁLISE DE REQUISITOS
}

\author{
Giani Petri, CAFW/UFSM, gianipetri@gmail.com \\ Natiel C. Chiavegatti, CAFW/UFSM, natiel.cazarotto@gmail.com
}

\begin{abstract}
Resumo. A engenharia de requisitos objetiva estabelecer o entendimento do problema e as necessidades do cliente. No entanto, o processo de ensino desta competência nem sempre é satisfatório, por ser um conteúdo teórico, muitos professores utilizam somente aulas expositivas, onde acaba limitando o aprendizado dos alunos. Assim, surge a necessidade de explorar novos métodos de ensino, como os jogos educacionais, de modo a criar um ambiente que desperte o interesse dos alunos. O objetivo deste trabalho é desenvolver e avaliar um jogo educacional do tipo RPG no qual o jogador atuará como um engenheiro de requisitos coletando e priorizando os requisitos em um cenário simulado. Uma avaliação sistemática realizada aponta resultados satisfatórios quanto a motivação, experiência do usuário e aprendizagem dos alunos.
\end{abstract}

Palavras - Chave: Engenharia de Requisitos, Jogo Educacional, RPG.

\section{A ROLE PLAYING GAME IN ELICITATION AND ANALYSIS OF REQUIREMENTS TEACHING}

\begin{abstract}
The requirements engineering objective to establish the understanding of the problem and the needs of the customer. However, the process of teaching this competence is not always satisfactory, as a theoretical content, many teachers use only lectures, which ends up limiting student learning. Thus, the need to explore new teaching methods, as educational games, to build an environment that will arouse the interest of students. The objective of this paper is to develop and evaluate a RPG educational game in which the player acts as a requirements engineer, collecting and prioritizing requirements in a simulated scenario. A systematic evaluation performed shows satisfactory results as motivation, user experience and student learning.
\end{abstract}

Keywords: Requirements Engineering, Educational Game, RPG.

\section{INTRODUÇÃO}

A Engenharia de Requisitos (ER) é uma das principais etapas dentro de um processo de desenvolvimento de um software. Objetiva estabelecer o entendimento entre o cliente o e engenheiro de software. No entanto, há pesquisas que apontam que a ER está sendo evidenciada como umas das principais causas de falhas em projetos, onde cerca de $85 \%$ dos problemas encontrados em softwares originam-se na etapa de Elicitação de Requisitos (GONÇALVES; THIRY; ZOUCAS, 2011), (FERNANDES; MACHADO; SEIDMAN, 2009). Isso pode ocorrer pelo fato de profissionais recém formados entrarem no mercado de trabalho sem possuir uma experiência prática de como exercer as atividades envolvidas nas etapas da engenharia de requisitos, em especial na etapa de elicitação e análise dos requisitos.

Em cursos de graduação, o ensino da competência de Engenharia de Requisitos, muitas vezes, deixa a desejar (THIRY; ZOUCAS; GONÇALVES, 2010). Pelo fato de ser um conteúdo bastante teórico, muitos professores enfatizam somente a teoria sem a 
aplicação prática, ou ainda, não utilizando estratégias de ensino diferenciadas, limitando assim o aprendizado dos alunos.

Neste contexto, o método de ensino predominante no ensino superior são as aulas expositivas (DJAJALAKSANA, 2011), onde o professor fala e os alunos ouvem. Esta estratégia é eficaz para contextualizar um assunto, principalmente, para grandes grupos de alunos. No entanto, pesquisas mostram que alunos ao participar destas aulas perdem o interesse e a concentração após dez a quinze minutos e começam a conversar com os colegas, enviar mensagens pelo celular e, até mesmo, entram em sonolência (BRABRAND, 2010). Além disso, este tipo de estratégia instrucional provoca apenas uma aprendizagem superficial no aluno (WAGNER, 1970).

Diante disso, surge a necessidade de explorar novos métodos de ensino para assim criar um aprendizado mais aprofundado nos alunos e potencializar cada vez mais o aprendizado, proporcionando um ambiente lúdico, interessante e prazeroso aos acadêmicos. Um dos recursos educativos disponíveis são os jogos educacionais, tendo por objetivo a contribuição com o aprendizado, explorando a simulação de atividades práticas e criando um ambiente que desperte interesse dos alunos. Um jogo educacional é composto basicamente por regras, restrições e um feedback informativo apresentando quais os seus erros e acertos no decorrer do jogo (WANGENHEIM; WANGENHEIM, 2012).

No entanto, os jogos existentes na literatura para o ensino de ER, em geral, objetivam apenas relembrar e recordar os conceitos aprendidos na aula expositiva, através de uma série de perguntas e respostas. Assim, acabam não aplicando na prática, através de uma simulação, os conceitos estudados em aula. Desta forma, este trabalho apresenta o desenvolvimento e a avaliação sistemática de um jogo educacional digital do tipo RPG (Role Playing Game), que simula um cenário real para o aluno colocar na prática os conceitos aprendidos na elicitação e análise de requisitos, utilizando a técnica de análise de cenários para a execução da atividade.

\section{METODOLOGIA}

A metodologia deste trabalho, quanto à natureza, classifica-se como uma pesquisa aplicada, onde objetiva-se gerar um produto de software (jogo educacional digital). Inicialmente, a pesquisa explorou artigos e livros publicados em bases acadêmicas (Google Scholar), como objetivo de identificar e analisar estudos sobre engenharia de requisitos, com um foco maior em elicitação de requisitos, quais seus métodos de ensino utilizando jogos digitais.

O presente trabalho será desenvolvido seguindo as seguintes etapas: estudos, pesquisas e análise de jogos digitais já existentes na área de engenharia de requisitos; escolha de uma ferramenta para o desenvolvimento do jogo; análise, projeto, desenvolvimento e avaliação sistemática do jogo educacional desenvolvido para o ensino de elicitação e análise de requisitos.

\section{TRABALHOS RELACIONADOS}

A revisão da literatura buscou jogos educacionais para o ensino de engenharia de requisitos nos seguintes mecanismos de buscas: Google Scholar(http://scholar.google.com.br/) e no IGR (Instrucional Games Repository) (http://srv.gqs.ufsc.br:8080/InstructionalGamesRepository/). Com os resultados da pesquisa os autores selecionaram três jogos para estudo e análise, considerando que os requisitos para a escolha foram adequação aos processos de engenharia de requisitos e 
ser um jogo educacional (digital ou não). Os jogos selecionados são: Ilha dos Requisitos (THIRY; ZOUCAS; GONÇALVES, 2010), SimSe (NAVARRO, 2006) e RE-O-Poly (GONÇALVES; THIRY; ZOUCAS, 2011), (SMITH; GOTEL, 2009).

O jogo Ilha dos Requisitos (THIRY; ZOUCAS; GONÇALVES, 2010) é um jogo em ambiente digital, cujo cenário é uma ilha. Sendo um jogo de perguntas e respostas, tem por objetivo o ensino dos princípios básicos de ER, disponibilizando dicas referentes as atividades que estão em andamento e um rápido feedback ao jogador, objetivando a compreensão dos processos de ER.

Por sua vez, o SimSe (NAVARRO, 2006) é um jogo digital onde o jogador atuará como um gerente de projetos. O SimSe aborda a Engenharia de Requisitos como uma das etapas do processo de desenvolvimento do software, no entanto, a ênfase do jogo é para a gestão do projeto.

O jogo Re-O-Poly (GONÇALVES; THIRY; ZOUCAS, 2011) é um jogo de tabuleiro que auxilia no aprendizado de iniciantes em ER, reforçando atividades recémvistas em aula, o jogo é baseado em perguntas de conhecimentos gerais e outras de conhecimentos específicos sobre ER (SMITH; GOTEL, 2009).

Os resultados da pesquisa demonstram que ainda há poucos jogos educacionais que exploram os conteúdos da área de Engenharia de Requisitos. Dois (Ilha dos Requisitos e RE-O-Poly) dos três jogos encontrados na literatura abordam especificamente a ER, no entanto, o jogo limita-se a retomar conceitos teóricos, através de perguntas e respostas. O terceiro jogo (SimSe) o jogador atua como um gerente de projetos atuando em um cenário real, no entanto, o jogo não possui foco na $E R$, abordando todas as etapas para a gestão de um projeto. Desta forma, justifica-se o desenvolvimento de um jogo educacional onde o jogador assuma um papel de Engenheiro de Requisitos e consiga simular na prática algumas etapas da ER, não somente relembrando conceitos teóricos, potencializando assim, o aprendizado aos alunos.

\section{ETAPAS DO DESENVOLVIMENTO DO EAREq-GAME}

\subsection{Análise do EAReq-Game}

Nessa seção define-se os objetivos de aprendizagem a serem alcançados pelo jogo, analisam-se quais as características do público alvo, modo de interação e estratégia instrucional.

Público alvo: O público alvo do jogo são alunos que estão frequentando os cursos superiores na área da computação, com uma faixa etária de não menos de 17 anos, trata-se de um público jovem e que possuem conhecimento sobre jogos.

Contexto organizacional: $\mathrm{O}$ jogo poderá ser aplicado no decorrer da disciplina de Engenharia de Software, mais especificamente nos processos de elicitação e análise de requisitos. Por ser um jogo digital, pode ser aplicado no decorrer da competência em sala de aula ou como tarefa de casa.

A aplicação da atividade (jogo): poderá ser realizada após a explicação dos conceitos de processo de elicitação e análise de requisitos.

Interação Jogo/Acadêmico: Modo de interação será de aprendizado individual, ondem apenas um acadêmico irá interagir como o jogo executando suas tarefas.

Estratégia Instrucional: A estratégia instrucional será de colocar em prática seus conhecimentos sobre elicitação e análise de requisitos aprendidos em teoria, por meio do jogo educacional. 
Objetivos de Aprendizagem: ( $i$ ) aplicar a elicitação de requisitos usando a técnica de análise de cenários; (ii) entender e aplicar a priorização dos requisitos coletados no cenário, classificando-os em obrigatório, desejável e fora do escopo.

\subsection{Projeto e Desenvolvimento do EAReq-Game}

Esta seção descreve sobre o funcionamento do jogo educacional digital EAReq-Game e as suas principais características. A Tabela 1 define a concepção do jogo EAReq-Game.

Tabela 1 - Concepção do EAReq-Game.

\begin{tabular}{c|c}
\hline Característica & Descrição \\
\hline Meta do Jogo & Auxiliar no ensino de engenharia de requisitos, com ênfase em \\
elicitação e análise de requisitos.
\end{tabular}

\subsubsection{Engine de implementação}

Para a criação do jogo foi utilizada a ferramenta RPG Maker VX (RPG MAKER, 2014), essa engine é utilizada para criação de jogos de RPG em ambientes 2D, por ser uma engine específica para desenvolvimento de atividade em RPG disponibiliza várias funcionalidades muito utilizadas como habilidades, efeitos, evolução, personagens, entre outras funções que podem ser utilizadas. O RPG Maker VX utiliza programação através de eventos, onde desenvolve-se apenas a lógica de programação e as mensagens que irão aparecer no decorrer do jogo, os demais efeitos são realizados através de eventos.

\subsubsection{Cenário e fases do jogo}

Ao iniciar o jogo o jogador Ralph se encontrará no nível um do jogo, no lado externo do Colégio. Ao ingressar no Colégio o jogador deverá ir para a Sala da Secretaria, onde deverá coletar os requisitos que estão disponíveis naquele ambiente, ao todo são oito requisitos nesta fase.

Cada requisito elicitado deverá ser classificado dentre as três alternativas, que são elas: obrigatório, desejável e fora do escopo. Um requisito obrigatório é 
imprescindível para o desenvolvimento do sistema, um requisito desejável é opcional para o sistema e um requisito fora do escopo, não deve ser considerado.

Como pode ser observado nas Figuras 1 e 2, o jogador coletou o requisito número 2 (Figura 1) e posteriormente deverá classificá-lo como um requisito obrigatório, desejável e fora do escopo (Figura 2).

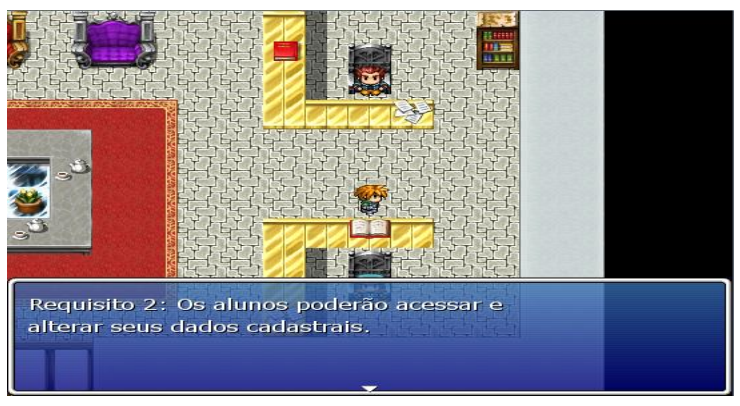

Figura 1 - Ralph coletando o requisito de número 2 da primeira fase do jogo EAReq-Game.

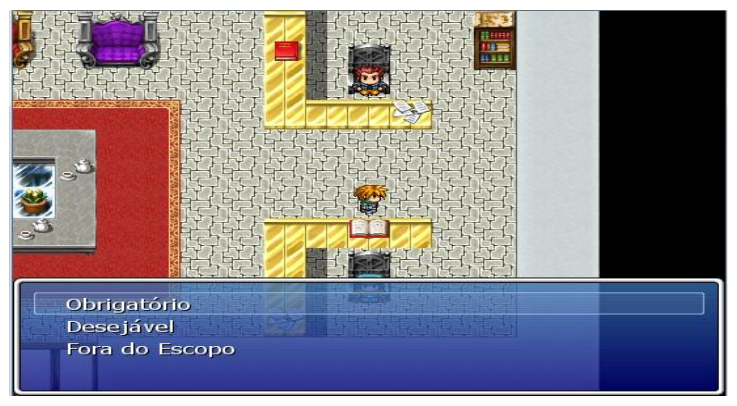

Figura 1 - Ralph priorizando o requisito 2 da primeira fase do jogo EAReq-Game.

Para cada classificação será acumulada uma pontuação conforme a corretude da resposta. Ao término de sua coleta de requisitos na sala da Secretaria o jogador deverá se dirigir até a saída da sala, onde receberá um feedback, que lhe informará quais foram os requisitos que ele priorizou corretamente e quais errou nessa fase. Após o feedback aparecerá uma mensagem informando se Ralph obteve sucesso ou não em sua primeira etapa da coleta de requisitos para o Sistema Acadêmico. Caso o jogador não obteve a pontuação mínima e não tiver mais nenhum requisito para coletar o jogador irá visualizar a tela de Game Over. Caso contrário, receberá uma mensagem para se dirigir até a sala da direção do colégio, pois obteve sucesso em sua elicitação e classificação de requisitos alcançando o número mínimo de pontuação exigido pela fase. A sala da direção corresponde a segunda fase do jogo.

De igual modo a primeira fase, na segunda fase o personagem Ralph também deverá interagir com objetos e outros personagens, para realizar a coleta e classificação dos requisitos, que estiverem disponíveis na sala da direção. Na segunda fase, a cada classificação dos requisitos coletados também será atribuída uma pontuação. Para o jogador vencer o jogo deverá atingir uma pontuação mínima, previamente definida pelos desenvolvedores, que corresponde ao mínimo aceitável de requisitos obrigatórios para o Sistema Acadêmico.

O jogador poderá a qualquer momento consultar sua pontuação atual do jogo nas propriedades do jogo. Ao final da coleta de requisitos da segunda fase, o jogador se dirige até a porta de saída da sala da direção, onde receberá um feedback, com os erros e acertos. Caso o número de pontuação mínima não for atingida e ainda há requisitos para serem coletados e classificados na sala, o jogador é informado e deverá retornar a sala e coletar e classificar os requisitos faltantes. Caso contrário, com todos requisitos coletados e classificados o jogador irá receber o resultado final do jogo, determinado pela sua pontuação acumulada nas duas fases.

A Figura 3 apresenta que o jogador Ralph conseguiu cumprir as suas missões no jogo EAReq-Game, concluindo com sucesso a elicitação e classificação dos requisitos para o desenvolvimento do Sistema Acadêmico.

Em contrapartida, se o jogador não conseguiu atingir a pontuação mínima na segunda fase do jogo ele receberá a mensagem informativa apresentada na Figura 4 e será direcionado ao Game Over, indicando que sua missão acabou sem sucesso e que o 
projeto de desenvolvimento do Sistema Acadêmico não poderá ser continuado, pois os requisitos não foram coletados e classificados com êxito.

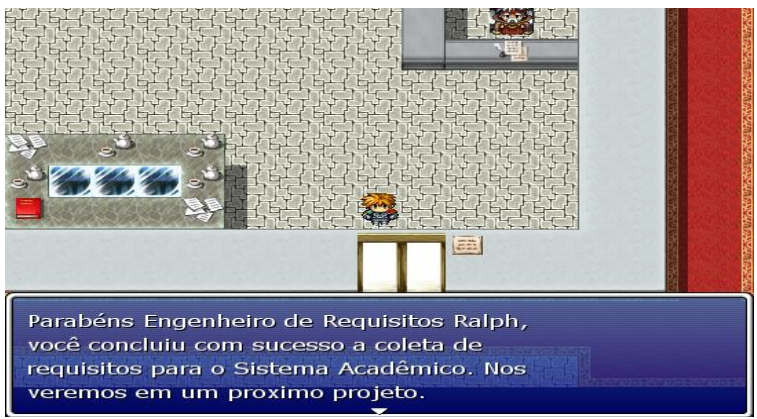

Figura 3 - Mensagem de final da segunda fase, obtenção de sucesso do jogo EAReq-Game.

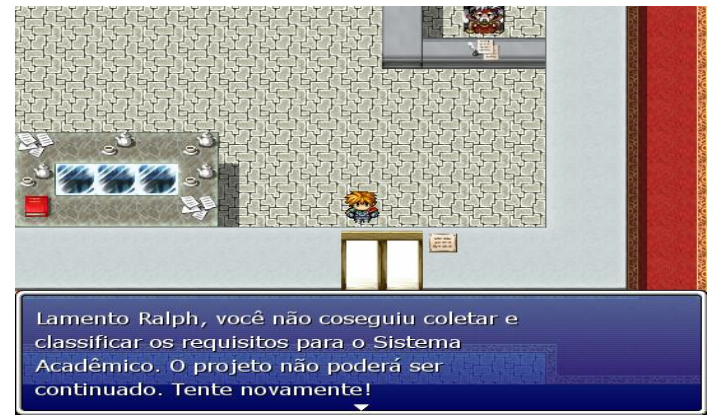

Figura 4 - Mensagem de final da segunda fase, não atingiu o objetivo no jogo EAReq-Game (Game Over).

\subsection{Avaliação do EAReq-Game}

O jogo EAReq-Game foi avaliado em termos de motivação, experiência do usuário e aprendizagem. A avaliação é baseada nos objetivos de aprendizagem definidos durante a etapa de análise e projeto (seção 5.1). O método de avaliação foi por meio de um estudo de caso utilizando o one-shot-post-test design. Neste método de avaliação os dados são coletados uma única vez, através de um questionário impresso, sendo que cada aluno recebeu um questionário para responder.

O questionário foi aplicado logo após os alunos realizarem a interação com o jogo educacional, nesse questionário o aluno responde questões referentes ao seu aprendizado após ter utilizando o jogo educacional. Para avaliação utiliza-se o modelo MEEGA de avaliações (SAVI, 2011).

A avaliação foi realizada no dia 24 de novembro de 2014 no Colégio Agrícola da Universidade Federal de Santa Maria (CAFW/UFSM), na cidade de Frederico Westphalen/RS. Para a realização desta avaliação utilizou-se um laboratório de informática do curso e um determinado tempo, foi realizado uma breve contextualização sobre a Engenharia de Requisitos e a etapa de Elicitação e Análise de Requisitos, além de uma contextualização sobre como seria realizada a avaliação, logo após os alunos interagiram com o jogo por determinado tempo. Ao todo participaram dessa avaliação 14 alunos.

Após a aplicação do jogo, foi entregue aos alunos um questionário de avaliação elaborado com base nas escalas do modelo MEEGA, onde os próprios alunos deveriam se auto avaliar, comparando seus conhecimentos antes do jogo e após a execução do jogo.

O questionário aplicado continha questões objetivas e questões descritivas. As questões descritivas eram de citar pontos fortes sobre o jogo e dar sua opinião sobre melhorias para o jogo, além de outras duas de auto avaliação, onde o próprio aluno atribuía uma nota sobre seus conhecimentos antes de ter vivenciado a experiência do jogo e após ter jogado.

As questões objetivas (que utilizam uma escala Likert) possuem 5 alternativas ($2,-1,0,+1,+2)$, onde o aluno deveria assinalar uma das alternativas, considerando que, "-2" o aluno discordasse fortemente com relação ao conteúdo das questões em relação ao jogo, e "+2" o aluno estaria com total concordância sobre o conteúdo das questões. O questionário aplicado aos alunos possuiu um total de, 27 questões objetivas e 4 questões 
descritivas, através das respostas foi possível gerar gráficos, onde representam as porcentagens de cada alternativa respondida pelo grupo de alunos.

Um dos componentes avaliados foram os termos de motivação do usuário ao interagir com o jogo, como podem ser visualizados os resultados no gráfico da Figura 5.

\section{Motivação}

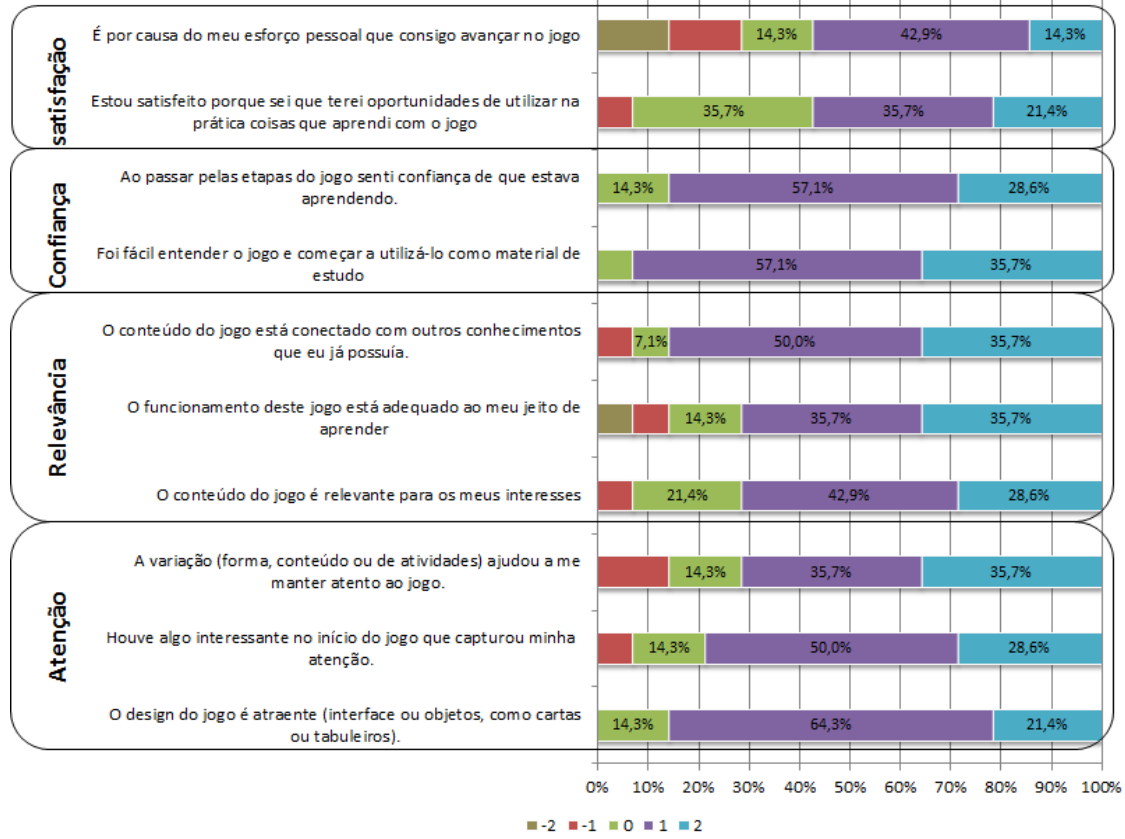

Figura 5 - Gráfico da escala de Motivação.

Observando o gráfico da Figura 5, pode-se perceber que a avaliação realizada pelos alunos aponta resultados significativamente positivos. Considerando que a maioria das questões está com índice de aprovação aproximadamente a 70\% nas alternativas +1 e +2 do questionário.

Ainda no gráfico da Figura 5, destacam-se algumas escalas como níveis de aprovação superiores a 70\%. Como é o caso da escala de confiança, que em sua primeira questão sendo ela: "Ao passar pelas etapas do jogo senti confiança de que estava aprendendo.", obteve $85,7 \%$ de respostas positivas (alternativas +1 e +2 ). Já a segunda questão "Foi fácil entender o jogo e começar a utilizá-lo como material de estudo", desta mesma escala, obteve nível de aprovação de 92,8\%.

$\mathrm{Na}$ escala de relevância destaca-se com maior nível de aprovação a questão: "O conteúdo do jogo está conectado com outros conhecimentos que eu já possuía.", obtendo nível positivo de $85,7 \%$. Na escala de atenção, a questão: “O design do jogo é atraente (interface ou objetos, como cartas ou tabuleiros).", obteve valores significativamente positivos, com $85,7 \%$ de respostas nas alternativas +1 e +2 .

Em termos de experiência do usuário ao interagir com o EAReq-Game, por meio dos dados coletados na avaliação, foi possível a apresentação dos resultados em um gráfico, disponível na Figura 6. 


\section{Experiência do Usuário}

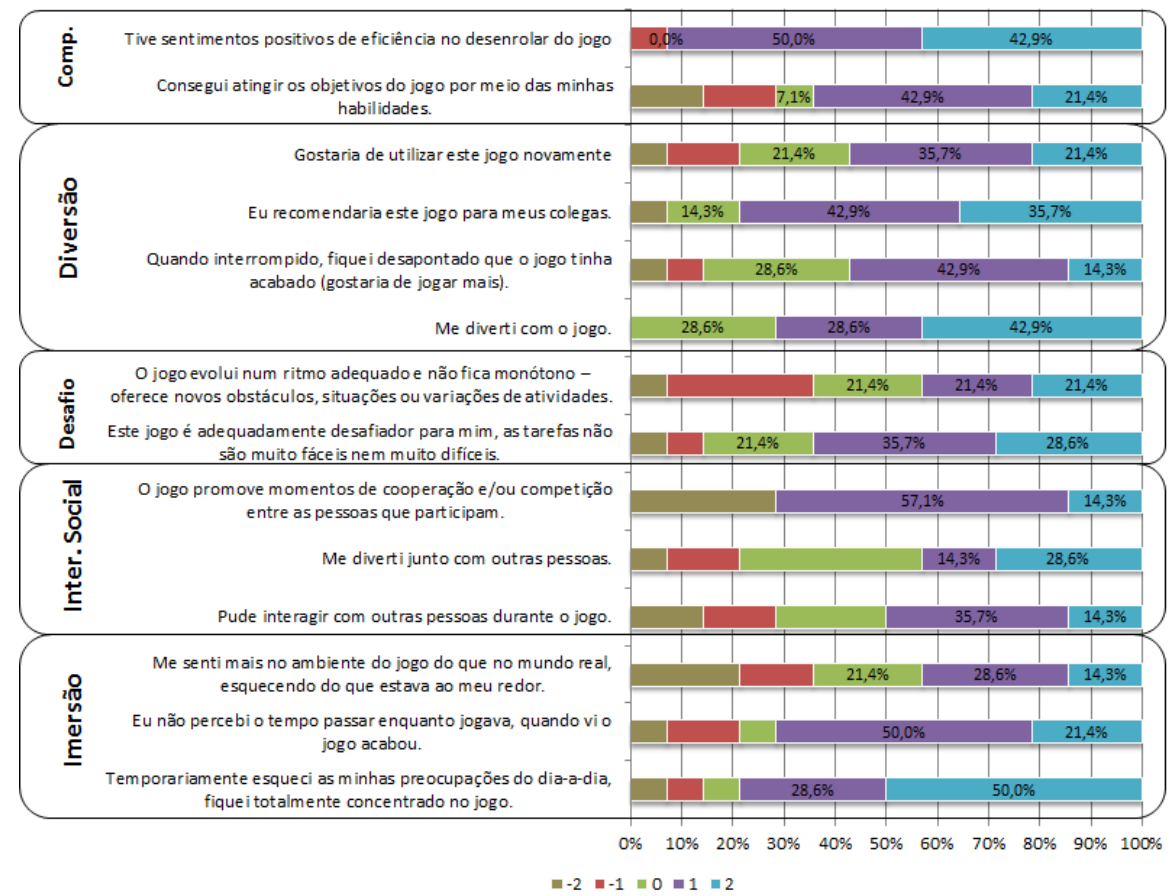

Figura 6- Gráfico que apresenta os resultados sobre a avaliação da experiência do usuário.

Como pode ser analisado no gráfico da Figura 6, foi possível obter valores positivos em praticamente todos os itens, exceto na escala de Interação Social, que seria a interação com outros usuários, porém como foi descrito no escopo do trabalho, esse jogo foi desenvolvido para apenas um único jogador e não uma competição entre alunos, logo, o resultado baixo desta questão é aceitável. Destacam-se as escalas de competência, imersão e diversão, onde foi possível identificar que o jogo atrai uma maior atenção do aluno para resolver as tarefas, exigindo suas habilidades e mantendo a confiança do aluno sobre o que estava fazendo.

Adicionalmente a aprendizagem também foi avaliada. Diante disso destaca-se um terceiro gráfico sobre a aprendizagem adquirida através do jogo, como pode ser observado na Figura 7.

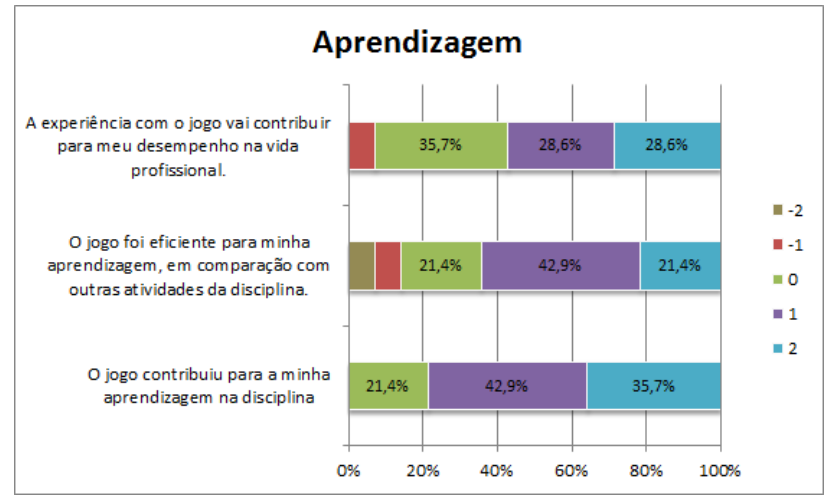

Figura 7 - Gráfico mostrando os resultados da avalição da aprendizagem do EAReq-Game.

Analisando o gráfico pode-se observar que, o jogo é uma boa estratégia para o ensino de elicitação e análise de requisitos, tendo em vista que a grande maioria dos 
resultados obtidos nesta avaliação, manteve-se concentradas nas alternativas $+1 \mathrm{e}+2$, onde aponta que a aprendizagem foi satisfatória no conteúdo abordado pelo jogo.

Conforme descrito anteriormente no questionário aplicado aos alunos, foram aplicadas duas questões de auto avaliação, sendo que os mesmos deveriam atribuir uma nota de 1 a 5, sendo 1 para "pouco conhecimento" e 5 para "muito conhecimento", para os conceitos de elicitação e análise de requisitos e priorização de requisitos, sobre qual era seu conhecimento antes do jogo ser aplicado e após a conclusão do jogo. Dessa forma, com as respostas a essas alternativas foi possível gerar um gráfico sobre o nível de conhecimento dos alunos, como apresentado nas Figuras 8 e 9.

A Figura 8 apresenta a média das notas da auto avaliação referente ao conceito de Elicitação e Análise de Requisitos. Os alunos precisavam se auto avaliar referente aos itens: lembrar o que é, compreender como funciona e aplicar na prática. Já a Figura 9 apresentam a média das notas da auto avaliação referente ao conceito de Priorização dos Requisitos. Os alunos também precisavam se auto avaliar referente aos itens: lembrar o que é, compreender como funciona e aplicar na prática.

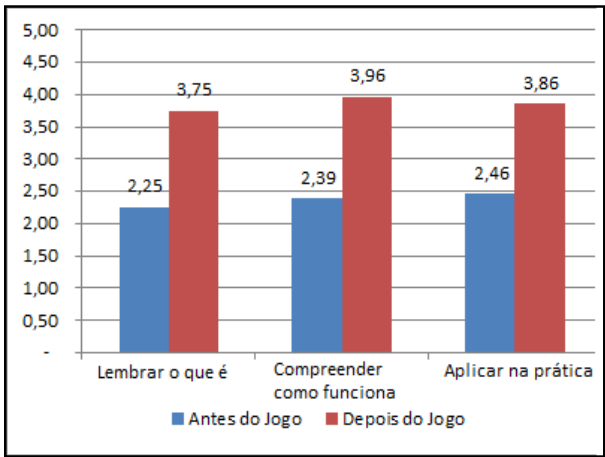

Figura 8 - Nível de conhecimento dos alunos antes e após a interação com o jogo.

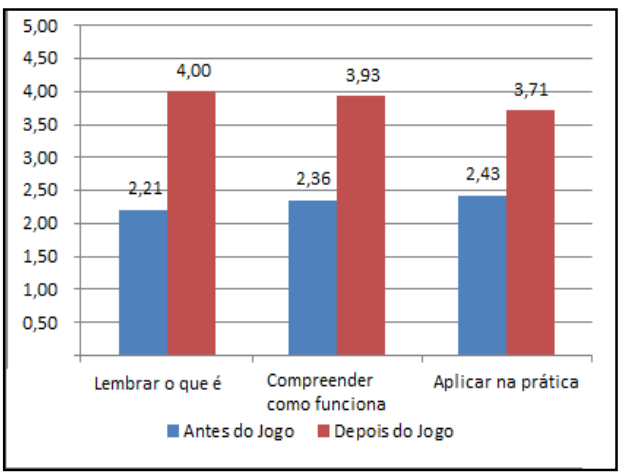

Figura 9 - Nível de conhecimento dos alunos antes e após a interação com o jogo.

Os gráficos (Figuras 8 e 9) apresentam claramente um acréscimo na aprendizagem da amostra de alunos participantes da avaliação, reforçando a ideia de que o jogo EAReq-Game alcançou seus objetivos em relação ao auxílio na aprendizagem da competência de elicitação e análise de requisitos.

\section{CONCLUSÃO}

Este trabalho tinha como objetivo o desenvolvimento e a avaliação de um jogo educacional digital do tipo RPG, para auxiliar no ensino da competência de elicitação e análise de requisitos, para cursos de graduação. Após ter desenvolvido o jogo educacional, realizou-se uma avaliação sistemática do jogo, baseada no modelo MEEGA. A avaliação apontou resultados satisfatórios. Concluindo que o jogo cumpriu com seus objetivos educacionais, sendo que cerca de $78 \%$, dos alunos em sua auto avaliação identificaram que o jogo contribuiu em seu aprendizado para a disciplina. Identificando assim que o jogo poderá servir de auxílio para o aprendizado da competência de elicitação e análise de requisitos.

Desta forma, conclui-se que os jogos educacionais contribuem ao aprendizado de competências em salas de aula, tendo em vista que buscam manter o aluno com foco e a atenção em suas tarefas, contribuindo assim com a aprendizagem, colocando em prática os seus conhecimentos adquiridos em aulas teóricas, sem colocar em risco nenhum projeto, pois apenas é um jogo que possui objetivos educacionais. 
Como trabalhos futuros, pretende-se analisar as sugestões de melhorias apontadas na avaliação e implementar as sugestões que são viáveis. As principais sugestões de melhorias apontadas foram: incluir mais fases para o jogo, mais desafios, aumentar o nível de dificuldade, entre outros.

\section{REFERÊNCIAS BIBLIOGRÁFICAS}

BRABRAND, C.; How to make sure your students learn what you want them to. Recife: UFPE, 2010. Disponível em: <http//www.itu.dk/people/brabrand/TeachingLearning-UFPE-2010.ppt.>

DJAJALAKSANA, Y. M.; A national survey of instructions strategies used to teach information systems courses: an exploratory investigation. $\mathrm{PhD}$ thesis, Florida, EUA: University of South Florida, 2011.

FERNANDES, J. M.; MACHADO, R. J.; SEIDMAN, S. B.; A requirements engineering and management training course for software development professionals. In: 22th CONFERENCE ON SOFTWARE ENGINEERING EDUCATION AND TRAINING. Hyderabad, Índia: IEE, 2009.

GONÇALVES, R. Q.; THIRY, M.; ZOUCAS, A.; Avaliação da aprendizagem em experimentos com jogos educativos de engenharia de requisitos. São José, SC: UNIVALI, 2011. Disponível em: <http://www.lbd.dcc.ufmg.br/colecoes/sbqs/2011/SBQS2011-TT14_82900_1.pdf>

NAVARRO, E.; SimSe: a software engineering simulation environment for software process education. California: University of California (IRVINE), 2006.

RPG MAKER. RPG Maker - Make your own Role-Playing-Games. Disponível em: <http://www.rpgmakerweb.com/> acesso em 15 de agosto de 2014.

SAVI, R.; Avaliação de jogos voltados para a disseminação do conhecimento. Florianópolis, SC: UFSC, 2011. Originalmente apresentado como Tese de Doutorado, Universidade Federal de Santa Catarina, 2011.

SMITH, R.; GOTEL, O.; RE-O-Poly: a customizable game to introduce and reinforce requirements engineering good practices. New York: Department of Computer Science, Pace University, 2009. Disponível em: 〈http://csis.pace.edu/ ctappert/srd2009/b4.pdf>

THIRY, M.; ZOUCAS, A; GONÇALVES, R. Q.; Promovendo a aprendizagem de engenharia de requisitos de software através de um jogo educativo. In: SIMPÓSIO BRASILEIRO DE INFORMÁTICA NA EDUCAÇÃO. João Pessoa: UFPB, 2010. Disponível em: <http://www.br-ie.org/pub/index.php/sbie/article/view/1514/1279>

WAGNER, R. W. Edgar Dale: professional theory into practice. Edgar Dale, p. 89-95, 1970.

WANGENHEIM, C. G. V; WANGENHEIM, A. V. Ensinando computação com jogos. Florianópolis: Bookes, 2012. 